\title{
Habilidades Sociais e Prevenção do Suicídio: Relato de Experiência em Contextos Educativos
}

\section{Social Skills and Suicide Prevention: Experience Report in Educational Contexts}

Habilidades Sociales y Prevención del Suicidio: Relato de Experiencia en Contextos Educativos

\author{
Vanessa Barbosa Romera Leme* \\ Universidade do Estado do Rio de Janeiro - UERJ, Rio de Janeiro, Rio de Janeiro, \\ Brasil
}

\section{Adriana Pinheiro Serqueira das Chagas**}

Universidade do Estado do Rio de Janeiro - UERJ, Rio de Janeiro, Rio de Janeiro, Brasil

Aline Penna-de-Carvalho***

Universidade do Estado do Rio de Janeiro - UERJ, Rio de Janeiro, Rio de Janeiro, Brasil

\section{Amanda Porto Padilha****}

Universidade do Estado do Rio de Janeiro - UERJ, Rio de Janeiro, Rio de Janeiro, Brasil

Ana Júlia de Carvalho Pereira Alves*****

Universidade do Estado do Rio de Janeiro - UERJ, Rio de Janeiro, Rio de Janeiro, Brasil

\section{Carolina Seixas da Rocha******}

Universidade do Estado do Rio de Janeiro - UERJ, Rio de Janeiro, Rio de Janeiro, Brasil

Fernanda de Azevedo França*******

Universidade do Estado do Rio de Janeiro - UERJ, Rio de Janeiro, Rio de Janeiro, Brasil

Fernanda dos Santos Quintanilha de J esus********

Universidade do Estado do Rio de Janeiro - UERJ, Rio de Janeiro, Rio de Janeiro, Brasil

Fernanda Pereira Calabar*********

Universidade do Estado do Rio de Janeiro - UERJ, Rio de Janeiro, Rio de Janeiro, Brasil 
Lana Pereira Mattos**********

Universidade do Estado do Rio de Janeiro - UERJ, Rio de Janeiro, Rio de Janeiro, Brasil

\title{
Letícia Costa Leopoldino*
}

Universidade do Estado do Rio de Janeiro - UERJ, Rio de Janeiro, Rio de Janeiro, Brasil

\section{Luana de Mendonça Fernandes***}

Universidade do Estado do Rio de Janeiro - UERJ, Rio de Janeiro, Rio de Janeiro, Brasil

\section{Priscila Sá da Silveira**}

Universidade do Estado do Rio de Janeiro - UERJ, Rio de Janeiro, Rio de Janeiro, Brasil

\begin{abstract}
RESUMO
Este estudo tem por objetivo apresentar um relato de experiência de um projeto que realizou oficinas e palestras sobre habilidades sociais e de vida em diferentes contextos educativos. As ações extensionistas são desenvolvidas com estudantes, docentes e funcionários técnicosadministrativos de uma universidade pública e alunos, professores e demais funcionários de escolas públicas da educação básica, localizadas no Estado do Rio de Janeiro. São apresentadas quatro oficinas e uma palestra realizadas na universidade e nas escolas. Todas as ações foram baseadas na promoção das habilidades sociais e de vida e evidenciaram, por meio dos relatos dos participantes, alguns ganhos no autoconhecimento e satisfação com atividades propostas. Ao final são analisados o impacto das atividades e os desafios que envolveram o projeto com foco na promoção de saúde mental e prevenção do suicídio.
\end{abstract}

Palavras-chave: habilidades sociais, habilidades de vida, suicídio, prevenção, saúde mental.

\section{ABSTRACT}

This study aims to present an experience report of a project that conducted workshops and lectures on social and life skills in different educational contexts. Extension actions are developed with students, teachers and technical-administrative staff of a public university and students, teachers and other employees of public primary schools, located in the State of Rio de Janeiro. Four workshops and a lecture are described in the university and schools. All actions were based on the promotion of social and life skills and showed, through the participants' reports, some gains in self-knowledge and satisfaction with proposed activities. At the end, the impact of the activities and the challenges involved in the project, focusing on the promotion of mental health and suicide prevention, are analyzed.

Keywords: social skills, life skills, suicide, prevention, mental health.

\section{RESUMEN}

Este estudio tiene por objetivo presentar un relato de experiencia de un proyecto que realizó talleres y conferencias sobre habilidades sociales y de vida en diferentes contextos educativos. Las acciones extensionistas se 
desarrollan con estudiantes, docentes y funcionarios técnicosadministrativos de una universidad pública y alumnos, profesores y demás funcionarios de escuelas públicas de educación básica, ubicadas en el Estado de Río de Janeiro. Se describen cuatro talleres y una conferencia en la universidad $y$ en las escuelas. Todas las acciones se basaron en la promoción de las habilidades sociales y de vida y evidenciaron, a través de los relatos de los participantes, algunas ganancias en el autoconocimiento y satisfacción con actividades propuestas. Al final se analizan el impacto de las actividades y los desafíos que involucraron el proyecto con foco en la promoción de salud mental y prevención del suicidio.

Palabras chave: habilidades sociales, habilidades de vida, suicídio, prevención, salud mental.

Embora o suicídio faça parte da história da humanidade, o tema ainda é tratado pela população em geral com estigma, preconceito, mitos e tabus (Schlösser, Rosa, \& More, 2014). Contudo, conforme especialistas da área, o suicídio deve ser compreendido como um fenômeno multifatorial e resultado de uma complexa interação de fatores biológicos, psicológicos e culturais presentes num determinado momento histórico (Bertolote, 2012). Atos e intenções suicidas não devem ser analisados como algo que tem uma fonte causal única e interna (genética/biológica) ao indivíduo (Cardoso et al., 2012). De acordo com a Organização Mundial da Saúde (OMS), mais de 800000 pessoas do mundo morreram, em 2016, devido ao suicídio. É a segunda principal causa de morte entre indivíduos de 10 a 24 anos e se caracteriza como um problema de saúde pública global (OMS, 2018). O Brasil é o oitavo país em número de suicídios e, em 2016, foram registradas 11.433 mortes, sendo que esse número representa um crescimento de $2,3 \%$ em relação ao ano anterior (OMS, 2018).

Nesse sentido, estudos (Braga \& Dell'Aglio, 2013; Oliveira, Bicalho, Teruel, Kahey, \& Botti, 2017; Schlösser et al., 2014) têm identificado, de modo sistemático, um conjunto de indicadores de risco pessoais, ambientais e sociais associados a maiores índices de atos/intenções suicidas com adolescentes e jovens, a saber: ideação suicida; tentativa prévia; transtorno mentais (depressão, ansiedade); impulsividade; rigidez cognitiva; uso de drogas lícitas/ilícitas; relações interpessoais conflituosas com familiares, pares e parceiros românticos, desesperança; exposição à violência na família (abuso físico e sexual) e na comunidade (bullying e homofobia); pobreza; percepção precária de suporte familiar e social; ausência de serviços públicos de saúde mental. Entretanto, conhece-se menos sobre indicadores de proteção individuais e contextuais do suicídio na transição para a vida adulta (Braga \& Dell' Aglio, 2013; Schlösser et al., 2014), sendo que, dentre eles, destacam-se: autoeficácia; autoestima; sentido de vida; habilidades sociais e de vida (empatia, comunicação eficaz, assertividade, lidar com estresse e resolução de 
problemas); integração e bom convívio com familiares, pares e parceiros românticos; religiosidade; percepção de apoio social da família, amigos e comunidade; acesso aos serviços públicos de saúde mental.

Pesquisas têm evidenciado que, na atualidade, os espaços educativos (como escolas e universidades) têm sido caracterizados por relações interpessoais individualistas e competitivas, práticas discriminatórias e pela "medicalização" dos processos de ensino e aprendizagem, isto é, as dificuldades relacionados à aprendizagem são tratadas muitas vezes com uso abusivo de medicamentos psicotrópicos, sem considerar aspectos relacionais, sociais e políticos (Chagas \& Pedrosa, 2016; Moretti \& Hubner, 2017). Essa conjuntura inviabiliza o surgimento de recursos protetores do suicídio, leva ao sofrimento psíquico, prejudica padrões de sociabilidade pautados por solidariedade e diminui o sentido de vida e o bem-estar geral da pessoa. Evidencia-se, portanto, a necessidade de não só prevenir o suicídio como também propiciar condições de promoção da saúde mental dessa população. Uma alternativa alinhada ao Plano de Ação em Saúde Mental 2015-2020, proposto pela Organização Pan Americana de Saúde (OPAS, 2016) para a prevenção do suicídio é investir em ações que promovam relacionamentos saudáveis e ampliem conhecimentos sobre diversidade sexual e ético-racial, questões de gênero e direitos humanos com a comunidade escolar e universitária. Uma maneira de atuar nessa direção é melhorar a qualidade das relações interpessoais por meio do desenvolvimento e ampliação das habilidades sociais e de vida. De fato, essas habilidades atuam no empoderamento dos adolescentes e jovens na medida em que facilitam o pensamento crítico e reflexivo, diminui práticas racistas, homofóbicas, sexistas e atitudes coercitivas/passivas (Del Prette \& Del Prette, 2017; Murta, Del Prette, $\&$ Del Prette, 2010)

\section{O projeto de extensão}

O Programa de Desenvolvimento Interpessoal para a Prevenção do Suicídio e Promoção de Saúde Mental no Curso de Vida (PRODIN) é um projeto de extensão que atua desde 2015, na Universidade do Estado do Rio de Janeiro. Tem por finalidade promover relações interpessoais positivas, contribuindo para a promoção de saúde mental e prevenção de indicadores de risco ligados à maior incidência de suicídio no curso de vida. O público-alvo das suas atividades (oficinas, cursos, palestras e intervenções) são estudantes, docentes e funcionários técnicos-administrativos da Universidade do Estado do Rio de Janeiro e alunos, professores, supervisores, orientadores e gestores de escolas públicas da educação básica. Todas as ações do 
projeto são embasadas na promoção de habilidades sociais e de vida dos participantes. Compõem o PRODIN alunos da graduação e pósgraduação, estando também vinculado ao programa UERJ pela VIDA, que visa por meio de uma equipe multidisciplinar desenvolver ações de prevenção ao suicídio. Assim, o presente estudo tem por objetivo apresentar um relato de experiência de um projeto que realizou oficinas e palestras sobre habilidades sociais e de vida em diferentes contextos educativos.

\section{Atividades extensionistas}

São apresentadas a seguir algumas atividades realizadas pelo PRODIN, no ano de 2018, a saber: três oficinas com estudantes na universidade e uma oficina e uma palestra realizada em escolas públicas. A literatura tem indicado que a promoção de habilidades sociais e de vida é uma via para a promoção de saúde mental e prevenção do suicídio (Feitosa, 2014; Murta et al., 2010). Assim, de modo a contribuir com algumas evidências para futuras intervenções nessa área, as ações foram avaliadas por meio de alguns instrumentos:

Ficha de inscrição. Elaborado pelas autoras, contém informações sobre as oficinas, tais como temas de cada encontro, dias, horários, número máximo de vagas e entrega de certificados além de questões referentes aos dados demográficos dos participantes, como nome, idade, sexo, e-mail, curso, período, acompanhamento psicológico e psiquiátrico e conhecimento prévio sobre habilidades sociais. Os dados obtidos nas fichas de inscrição foram inseridos em uma planilha do programa Microsoft Excel para a caracterização da amostra.

Protocolo para avaliação da qualidade de implementação. Desenvolvido por Murta (2017) para investigar indicadores de processo da qualidade de implementação dos programas. É organizado em quatro partes que avaliam a fidelidade, o desempenho do facilitador, indicadores de apego e adesão dos participantes. No presente estudo foi aplicado nas oficinas e utilizado os itens que descrevem os comportamentos dos participantes de busca de proximidade com o facilitador e adesão nas atividades. Contém uma lista de 15 comportamentos verbais e não verbais, como por exemplo, fazer perguntas pessoais e olhar com atenção. A ocorrência desses comportamentos é observada e registrada durante cada atividade, independentemente de quem apresentou os comportamentos. Ao final das oficinas, as cofacilitadoras discutiam sobre quais comportamentos do protocolo haviam sido manifestos pelos participantes. Assim, quando a dupla de cofacilitadoras estava de acordo, a presença ou ausência dos comportamentos eram 
anotadas de maneira global, independentemente da quantidade de manifestações pelos participantes. Posteriormente, foram somadas as frequências de cada comportamento ao final de todas as oficinas e calculado a porcentagem, considerando o total de ocorrências possíveis.

Avaliação do impacto imediato da sessão. Desenvolvido por Murta (2008) para investigar a satisfação do participante com a sessão e as descobertas assimiladas. Dispõe de uma lista de sentimentos, pensamentos e comportamentos positivos (por exemplo, "Me senti relaxado") e negativos ("Fiquei tenso") que podem surgir enquanto a atividade está em andamento. A avaliação é constituída por 23 itens distribuídos em uma escala tipo Likert de 1 ("Não aconteceu comigo") a 3 ("Aconteceu bastante comigo"). No presente estudo, esse instrumento foi utilizado nas oficinas e palestra e a sua pontuação foi modificada para uma escala que variou de 0 a 2. Em seguida, os resultados foram tabulados no programa Microsoft Excel, no qual, calculou-se a frequência dos sentimentos, comportamentos e pensamentos positivos e negativos para cada participante ao final de cada atividade.

Adaptação da dinâmica "Que bom, que pena e que tal". Desenvolvida por Berkenbrock (2014) para investigar a satisfação dos participantes após o encerramento da oficina e palestra nas escolas. Compreende frases disparadoras "Que bom", "Que pena" e "Que tal", que procuram identificar, respectivamente, os aspectos positivos, negativos e sugestões a partir de respostas abertas, escritas individualmente pelos participantes, que foram transcritas literalmente numa planilha do programa Microsoft Excel. Posteriormente, as falas dos participantes foram categorizadas por dois pesquisadores como positivas, negativas ou sugestão. Outros dois pesquisadores atuaram como juízes na análise dessas categorias e, ao final, essas mesmas categorias foram quantificadas em frequências.

As oficinas com os estudantes universitários. Foram realizadas três oficinas que focaram, cada uma, algumas habilidades sociais e de vida: emoções e autoconhecimento; empatia; assertividade. Essas ações foram oferecidas para todos os cursos da Universidade do Estado do Rio de Janeiro e divulgadas por meio de redes sociais e por cartazes espalhados na universidade. Foi disponibilizado um link com acesso a ficha de inscrição on-line. Para participar das oficinas os universitários deveriam ser estudantes de qualquer curso da graduação na Universidade. Ao final das oficinas cada participante recebeu por e-mail um certificado, com carga horária de duas horas e os que participaram das três oficinas, receberam um certificado extra de seis horas. Inscreveram-se 85 estudantes, sendo que desses 24 participaram das oficinas: 18 do sexo feminino e seis do sexo masculino, com idade entre 18 e 59 anos ( $M=25,2$ anos). Do total, 
18 eram do curso de psicologia, três de pedagogia, dois de nutrição e um de geografia. A maioria dos participantes cursava do primeiro ao quarto período $(\mathrm{N}=13 ; 54,1 \%)$, seguido dos períodos medianos e finais do quinto ao $11^{\circ}$ período $(\mathrm{N}=11 ; 45,8 \%)$. Dos inscritos, $50,6 \%$ informaram que não faziam acompanhamento psicológico e 83,5\% estavam em acompanhamento psiquiátrico. A maioria não tinha conhecimento prévio sobre habilidades sociais $(N=53 ; 62,4 \%)$. As três oficinas ocorrem nos meses de maio, junho e julho e cada uma teve duração de duas horas. O referencial teórico-metodológico das técnicas utilizadas nas oficinas foi Teoria Cognitivo-Comportamental. Assim, os procedimentos de ensino envolveram exposição dialogada, debates em pequenos grupos, vivências ("Olhos nos Olhos", "Reconhecendo e comunicando as emoções"; "Nem passivo nem agressivo" adaptadas de Del Prette \& Del Prette (2002), role playing e uso de recursos audiovisuais, tais como músicas (por exemplo, "Não precisa mudar", de Ivete Sangalo e Saulo Fernandes e "New rules", de Dua Lipa) e vídeos (por exemplo, trechos do filme "Minha mãe é uma peça", de Paulo Gustavo e André Pellenz). Durante todas as oficinas foi entregue uma cartilha para cada participante, confeccionada com conteúdo sobre o campo teórico e prático das habilidades sociais (Del Prette \& Del Prette, 2017). O objetivo do material foi gerar a reflexão dos participantes sobre seus contextos, estimulando-os a pensar sobre seus sentimentos, comportamentos e ações. Na última página de cada cartilha foi disponibilizado os números de telefones de clínicas psicológicas, emergenciais e não emergenciais. Cada oficina foi conduzida por dois facilitadores (discentes da pós-graduação) e em média dois cofacilitadoras (estudantes da graduação).

A primeira oficina (emoções e autoconhecimento) teve como proposta reconhecer sobre contextos geradores de emoções/sentimentos e refletir sobre as estratégias utilizadas na expressão emocional e no enfrentamento de situações identificadas pelos estudantes como estressantes e conflituosas em diversos contextos, tais como na família, universidade e emprego. Participaram 16 estudantes de graduação, sendo 11 do sexo feminino $(68,7 \%)$. A Avaliação de impacto imediato apresentou de um total de 416 pontos, um escore de $300(72,1 \%)$ para os aspectos positivos. Os itens mais frequentes foram "Tive confiança do grupo" $(n=30)$ e "Me senti com mais coragem para enfrentar certos problemas" $(n=26)$. Em relação aspectos negativos, foram computados 45 pontos $(14,1 \%)$, de um total de 320 , sendo que os itens mais relatados foram "Percebi que cuido mais dos outros do que de mim mesmo" $(n=14)$ e "Fiquei tenso" $(n=8)$.

$\mathrm{Na}$ segunda oficina (empatia), propuseram-se discussões críticas sobre expressar empatia ao adotar a perspectiva do outro, além de interpretar e compreender sentimentos e pensamentos próprios e dos 
interlocutores. Participaram 17 graduandos, sendo 11 do sexo feminino (64,7\%). A análise da Avaliação de impacto imediato dessa oficina mostrou $350(79,1 \%)$ escores positivos, de 442 pontos e 51 (15\%) escores negativos, de um total de 340 pontos. Os itens mais frequentes em relação aos aspectos positivos foram "Tive confiança no grupo" $(n=33)$ e "Me senti confiando mais em mim mesmo" $(n=28)$ e negativos foram "Percebi que cuido mais dos outros do que de mim mesmo" $(n=18)$ e "Tive sentimentos de culpa" $(n=8)$. Por fim, a terceira oficina (assertividade) teve por objetivo possibilitar reflexões críticas sobre reconhecer e diferenciar os desempenhos sociais assertivos, passivos e agressivos, identificar as consequências para si e para os outros, lidar com pedidos abusivos e propor estratégias individuais e coletivas frente às situações em que ocorriam violação de direitos humanos. Nessa oficina, compareceram 11 alunos de graduação, sendo sete do sexo feminino (63,3\%). Os escores da Avaliação de impacto atingiram uma pontuação de 235 $(82,1 \%)$ de um total de 286 pontos para os sentimentos, comportamentos e pensamentos positivos e os negativos somaram $38(17,2 \%)$, de um total de 220 pontos. Os aspectos positivos mais relatados foram "Me compreendi um pouco mais" $(n=33)$ e "Descobri algo sobre o que fazer para lidar com meus problemas" $(n=32)$ e os negativos foram "Fiquei tenso" $(n=21)$ e "Percebi que cuido mais dos outros do que de mim mesmo" $(n=19)$.

Ao final das oficinas foi realizada uma avaliação oral para que os participantes pudessem dar seus feedbacks sobre as atividades e relatar como se sentiram: "Essa oficina específica, com certeza vou levar por toda minha vida. Pois venho aprendendo a lidar com as minhas emoções e saber me colocar quando algo não me agrada"; "Eu sou uma pessoa agressiva e com a oficina me fez refletir melhor sobre o conceito e saber me controlar melhor"; "A partir desta oficina pude compreender melhor a relação abusiva que me encontrava". Em relação ao Protocolo de avaliação de processo, os resultados sinalizaram de um total de 15 comportamentos que indicaram confiança entre os participantes e entre esses e os facilitadores, nove $(60 \%)$ na primeira oficina e dez $(66,66 \%)$ na segunda e na terceira oficina. Os aspectos mais sinalizados pelos participantes foram relatar problemas, sentimentos e progressos pessoais, explicar as causas do próprio comportamento, falar sobre a vida pessoal fora da oficina, despedir e olhar com atenção.

\section{Palestra e a oficina nas escolas}

Além das três oficinas com os universitários, foram realizadas uma palestra (mês de maio) e uma oficina (mês de outubro), com duração de duas horas cada, em escolas públicas de Ensino Fundamental e 
Médio, localizadas no Estado do Rio de Janeiro. O referencial teóricometodológico das técnicas utilizadas nas oficinas foi Teoria CognitivoComportamental. Os recursos utilizados para realização das atividades foram a apresentação de conteúdos por meio de slides, questionamentos sobre o tema foco do encontro, com o intuito de gerar reflexões e pensamento crítico nos participantes, realização de exercícios em pequenos grupos para avaliar a compreensão dos conceitos apresentados e vivências.

A palestra foi realizada com alunos e professores dos anos finais do Ensino Fundamental de uma escola estadual, localizada em um município no Estado do Rio de Janeiro. Essa atividade teve por objetivo abordar algumas habilidades sociais e de vida (empatia e assertividade), aspectos relacionados à promoção de relações interpessoais positivas e à prevenção do suicídio no contexto escolar. Participaram 81 estudantes do 8 o ano e 9o ano. Desses, 29 responderam aos instrumentos que procuraram avaliar a atividade, sendo 21 do sexo feminino $(72,41 \%)$, com idade entre 13 e 18 anos $(\mathrm{M}=15,1$ anos). Além disso, participaram quatro professores. Os resultados da análise da Avaliação de impacto imediato indicaram 379 relatos positivos, de um total de 870 , o que corresponde a $44 \%$, com destaque para "Desejei realizar projetos de vida" $(n=38)$ e "Percebi que tenho forças para viver" $(n=35)$. Em relação aos sentimentos, comportamentos e pensamentos negativos, foram pontuados 103 (30\%) de um total de 348 escores negativos, sendo os mais frequentes "Me senti distraído e "voando" em alguns momentos" $(n=27)$ e "Percebi que cuido mais dos outros do que de mim mesmo" $(n=24)$. No que se refere aos resultados do instrumento "Que bom, que pena e que tal", foram evidenciados 80 relatos no total, sendo 44 (55\%) categorizados como positivos ("Gostei da iniciativa de trazer uma palestra com um assunto tão tabu", "Ajudou a mostrar as pessoas que algumas pessoas precisam de ajuda", menina, 14 anos). Foram registradas 23 (28,75\%) sugestões ("Ter palestras diferentes uma vez por mês", menina, 15 anos), "Ter esse projeto em toda escola do Brasil", jovem, 18 anos) e $13(16,25 \%)$ relatos categorizados como negativos ("Nem todo mundo participou", menina, 15 anos).

A oficina foi realizada com funcionários de uma escola federal, localizada na cidade do Rio de Janeiro. O objetivo da ação foi refletir sobre a cotidiano escolar e as práticas que contribuem para a saúde mental e sofrimento psíquico, fatores de risco e proteção associados ao suicídio na adolescência e transição para a vida adulta. Além disso, procurou-se ampliar os conhecimentos sobre as habilidades sociais e de vida (empatia e assertividade) que ocorrem nas relações interpessoais na escola. Participaram dessa atividade 22 funcionários, sendo $16(72,72 \%)$ do sexo feminino, com idades entre 28 e 59 anos $(\mathrm{M}=38,1$ anos). Dentre os participantes, seis eram orientadores 
educacionais/pedagógicos, quatro eram auxiliares/técnicos administrativos, três professores, três bibliotecários/auxiliares de biblioteca, dois psicólogos, um assistente social, um pedagogo, um técnico de laboratório e um não especificou o cargo. Os resultados da análise da Avaliação de impacto imediato indicaram 334 escores de sentimentos, comportamentos e pensamentos caracterizados como positivos de um total de 660, o que corresponde a 51\%, com destaque para "Me senti confiando mais em mim mesmo" $(n=31)$. Em relação aos relatos negativos, foram contabilizadas 45 (17\%) respostas de um total de 264, sendo o aspecto mais frequente "Percebi que cuido mais dos outros do que de mim mesmo" $(n=24)$. Quando analisado o instrumento de Avaliação da atividade "Que bom, que pena e que tal", registrou-se 40 relatos, sendo 21 (53\%) positivos ("Poder refletir sobre meus próprios sentimentos e emoções.", mulher, 38 anos), "Pude ver que as pessoas poderiam ser mais empáticas.", mulher, 29 anos), 13(33\%) de sugestões ("Buscar atividades contextualizadas com o cenário escolar.", homem, 30 anos), "Aprofundar o trabalho direto com os estudantes.", mulher, 41 anos) e seis ( $15 \%$ ) negativos ("Termos pouco tempo para refletirmos mais sobre estratégias de ação.", mulher, 38 anos), "O tempo de discussão foi curto para o nosso grupo. Surgiram questões ricas que foram trabalhadas muito rapidamente e que poderiam ser aprofundadas caso houvesse mais tempo.", homem, 30 anos).

\section{Refletindo sobre a experiência}

De modo geral, as atividades tiveram bastante procura e participação. Destaca-se que três universitários participaram de todas as oficinas e 12 de duas, o que indica uma boa assiduidade, além de ter favorecido a promoção das habilidades sociais e de vida focalizadas. De modo geral, os relatos dos participantes evidenciaram alguns ganhos no autoconhecimento e satisfação com as oficinas e palestra, busca por estratégias coletivas para enfrentar de maneira assertiva situações opressoras nos espaços educativos, ampliação de conhecimentos sobre habilidades sociais e de vida e desenvolvimento do pensamento crítico e reflexivo. Dentre os desafios que atravessaram esse projeto, destaca-se que o suicídio é o desfecho final de um processo que envolve as múltiplas e dinâmicas interrelações entre a pessoa e seus contextos mais próximos tais como, a família, a comunidade e escola/universidade/trabalho, e distais, tais como políticas públicas, valores religiosos e práticas culturais acerca de papéis de gênero e orientação sexual e aspectos éticos-raciais (Bertolete, 2012, Schlösser et al., 2014). Desse modo, as ações propostas no projeto de extensão, baseado na promoção de algumas habilidades sociais e de vida, focaram alguns aspectos 
pessoais e relacionais (proximais), comumente associados a prevenção do suicídio e promoção de saúde mental e isso não esgota as possibilidades de intervenções. Outras propostas para a prevenção do suicídio devem incluir o acesso a tratamentos especializados em saúde mental nos serviços públicos e políticas para reduzir uso nocivo de drogas e álcool (OPAS, 2016).

Somado a isso, os programas de habilidades sociais seguem um racional (número de encontros, procedimentos de ensino etc.) e são, de modo geral, implementados por meio de delineamentos robustos, com avaliações de processo e pré-teste e pós-teste e grupo controle (Del Prette \& Del Prette, 2017). Assim, as ações extensionistas relatadas não tiveram como proposta seguir o rigor metodológico típico das intervenções em habilidades sociais, mas servir como uma alternativa complementar e adaptada a realidade dos contextos educativos que foram alvo do projeto. Nesse sentido, as atividades facilitaram a difusão dos conhecimentos do campo das habilidades sociais para a comunidade, contribuindo para que outras ações, como intervenções possam ser futuramente implementadas com adesão. De fato, é necessário a ampliação de espaços de discussão sobre as relações interpessoais que causam sofrimento psíquico em escolas e universidades. Entende-se que as habilidades sociais e de vida poderão servir como ferramentas de empoderamento de estudantes, professores e demais profissionais da área da educação, como também na diminuição de práticas sociais discriminatórias. Por fim, espera-se que o relato dessa experiência possa influenciar futuros estudos de prevenção do suicídio e promoção em saúde mental nos contextos educativos.

\section{Referências}

Bertolote, J. M. (2012). O suicídio e sua prevenção. São Paulo: Ed. Unesp.

Braga, L. L., \& Dell'Aglio, D. D. (2013). Suicídio na adolescência: Fatores de risco, depressão e gênero. Contextos Clínicos, 6(1), 2-14. doi: 10.4013/ctc.2013.61.01

Berkenbrock, V. J. (2014). Dinâmicas para encontros de grupo: Para apresentação, intervalo, autoconhecimento e conhecimento mútuo, amigo oculto, despertar, avaliação e encerramento. Petrópolis, RJ: Editora Vozes.

Cardoso, H. F., Baptista, M. N., Ventura, C. D., Branão, E. M., Padovan, F. D., \& Gomes, M. A. (2012). Suicídio no Brasil e América Latina: revisão bibliométrica na base de dados Redalycs. Diaphora, 12(2), 42-48. Recuperado de http://www.sprgs.org.br/diaphora/ojs/index.php/diaphora/articl e/view/69 
Chagas, J. C., \& Pedroza, R. L. S. (2017). Patologização e medicalização da educação superior. Psicologia: Teória e Pesquisa, 32(1), 1-10. doi: 10.1590/0102-3772e32ne28

Del Prette, A., \& Del Prette, Z. A. P. (2002). Psicologia das relações interpessoais e habilidades sociais: Vivências para o trabalho em grupo. Petrópolis: Vozes.

Del Prette, A., \& Del Prette, Z. A. P. (2017). Competência social e habilidades sociais: Manual teórico-prático. Petrópolis: Vozes.

Feitosa, F. B. (2014). A depressão pela perspectiva biopsicossocial e a função protetora das habilidades sociais. Psicologia: Ciência e Profissão, 34(2), 488-499. doi: 10.1590/1982-3703000992013

Moretti, F. A., \& Hübner, M. M. C. (2017). O estresse e a máquina de moer alunos do ensino superior: Vamos repensar nossa política educacional? Revista de Psicopedagogia, 34(105), 258-267. Recuperado http://pepsic. bvsalud.org/pdf/psicoped/v34n105/03.pdf

Murta, S. G. (2017). Aproximando ciência e comunidade: Difusão de programas de habilidades sociais baseados em evidencias. In A. Del Prette, \& Z. A. P. Del Prette (Orgs.), Habilidades sociais: Intervenção efetivas em grupo (2a ed., pp. 83-111). São Paulo: Casapsi Livraria e Editora Ltda.

Murta, S. G., Del Prette, A., \& Del Prette, Z. A. (2010). Prevenção ao sexismo e ao heterossexismo entre adolescentes: Contribuições do treinamento em habilidades de vida e habilidades sociais. Revista de Psicologia da Criança e do Adolescente, 1(2), 73-87. Recuperado de http://revistas.lis.ulusiada. pt/index. php/rpca/article/view/21/pd $f$

Murta, S. G. (2008). A prática grupoterápica sob enfoque psicoeducativo: uma introdução. In S. G. Murta (Org.), Grupos Psicoeducativos: Aplicações em múltiplos contextos (pp. 1328). Goiânia: Porã Cultural.

Schlösser, A., Rosa, G. F. C., \& More, C. L. O. (2014). Comportamento suicida ao longo do ciclo vital. Temas em Psicologia, 22(133), 135-145. doi: 10.9788/TP2014.1-11

Oliveira, A. M., Bicalho, C. M. S., Teruel, F. M., Kahey, L. L., \& Botti, N. C. L. (2017). Comportamento suicida entre adolescentes: Revisão integrativa da literatura nacional. Adolescência \& Saúde, 14(1), 88-96. Recuperado de http: //www.adolescenciaesaude.com/detalhe_artigo. asp?id=63 9

Organização Mundial da Saúde [OMS]. (2018). World Health Statistics 2018. Genebra: Organização Mundial da Saúde. Recuperado de https: //apps. who.int/iris/bitstream/handle/10665/272596/9789 241565585-eng. pdf?ua $=1 \& u a=1$ 
Organização Pan Americana de Saúde [OPAS]. (2016). Plan of action on mental health 2015-2020. Genebra: Organização Mundial da Saúde. https://www. paho.org/hq/index. php?option=com content\&view $=$ article\&id =11337: plan-of-action-on-mental-health-2015$2020 \&$ lemid $=41600 \&$ lang $=f$

\section{Endereço para correspondência Vanessa Barbosa Romera Leme}

Universidade do Estado do Rio de Janeiro - UERJ

Rua Francisco Xavier, 524, 10 andar, bl. B sl. 10019, Maracanã, CEP 20550-900, Rio de Janeiro - RJ, Brasil

Endereço eletrônico: vanessaromera@gmail.com

Adriana Pinheiro Serqueira das Chagas

Universidade do Estado do Rio de Janeiro - UERJ

Rua Francisco Xavier, 524, 10 andar, bl. B sl. 10019, Maracanã, CEP 20550-900, Rio de Janeiro - RJ, Brasil

Endereço eletrônico: drikaserqueira@hotmail.com

Aline Penna-de-Carvalho

Universidade do Estado do Rio de Janeiro - UERJ

Rua Francisco Xavier, 524, 10 o andar, bl. B sl. 10019, Maracanã, CEP 20550-900, Rio de Janeiro - RJ, Brasil

Endereço eletrônico: alinepennadecarvalho@gmail.com

\section{Amanda Porto Padilha}

Universidade do Estado do Rio de Janeiro - UERJ

Rua Francisco Xavier, 524, 10 andar, Bloco B, sala 10019, Maracanã, CEP: 20550-

900, Rio de Janeiro, RJ , Brasil

Endereço eletrônico: amandaportopadilha@gmail.com

\section{Anna J úlia de Carvalho Pereira Alves}

Universidade do Estado do Rio de Janeiro - UERJ

Rua Francisco Xavier, 524, 10 andar, bl. B sl. 10019, Maracanã, CEP 20550-900, Rio de Janeiro - RJ, Brasil

Endereço eletrônico: anajualves@gmail.com

\section{Carolina Seixas da Rocha}

Universidade do Estado do Rio de Janeiro - UERJ

Rua Francisco Xavier, 524, 10ㅇandar, bl. B sl. 10019, Maracanã, CEP 20550-900, Rio de Janeiro - RJ, Brasil

Endereço eletrônico: carolinaseixasrocha@gmail.com

\section{Fernanda de Azevedo França}

Universidade do Estado do Rio de Janeiro - UERJ

Rua Francisco Xavier, 524, $10^{\circ}$ andar, bl. B sl. 10019, Maracanã, CEP 20550-900, Rio de Janeiro - RJ, Brasil

Endereço eletrônico: fernandafranca.coordenadora@gmail.com

Fernanda dos Santos Quintanilha de J esus

Universidade do Estado do Rio de Janeiro - UERJ

Rua Francisco Xavier, 524, 10 andar, bl. B sl. 10019, Maracanã, CEP 20550-900, Rio de Janeiro - RJ, Brasil

Endereço eletrônico: quintanilha.fernandaa@gmail.com

Fernanda Pereira Calabar

Universidade do Estado do Rio de Janeiro - UERJ

Rua Francisco Xavier, 524, 10 andar, bl. B sl. 10019, Maracanã, CEP 20550-900, Rio de Janeiro - RJ, Brasil 
Endereço eletrônico: nandacalabar@yahoo.com.br

Lana Pereira Mattos

Universidade do Estado do Rio de Janeiro - UERJ

Rua Francisco Xavier, 524, 10 andar, bl. B sl. 10019, Maracanã, CEP 20550-900,

Rio de Janeiro - RJ, Brasil

Endereço eletrônico: lanamattosp@gmail.com

Letícia Costa Leopoldino

Universidade do Estado do Rio de Janeiro - UERJ

Rua Francisco Xavier, 524, $10^{\circ}$ andar, bl. B sl. 10019, Maracanã, CEP 20550-900,

Rio de Janeiro - RJ, Brasil

Endereço eletrônico: leticialeerj@hotmail.com

Luana de Mendonça Fernandes

Universidade do Estado do Rio de Janeiro - UERJ

Rua Francisco Xavier, 524, 10ำ andar, bl. B sl. 10019, Maracanã, CEP 20550-900,

Rio de Janeiro - RJ, Brasil

Endereço eletrônico: luapsi@gmail.com

\section{Priscila Sá da Silveira}

Universidade do Estado do Rio de Janeiro - UERJ

Rua Francisco Xavier, 524, 10 andar, bl. B sl. 10019, Maracanã, CEP 20550-900, Rio de Janeiro - RJ, Brasil

Endereço eletrônico: priscilasadasilveira@gmail.com

Recebido em: 16/01/2019

Reformulado em: 10/04/2019

Aceito em: 11/04/2019

\section{Notas}

* Professora adjunta do Instituto de Psicologia da UERJ e professora do Programa de Pós-Graduação em Psicologia da UERJ .

** Mestranda do Programa de Pós-Graduação em Psicologia da UERJ.

*** Doutoranda do Programa de Pós-Graduação em Psicologia da UERJ .

**** Discente no curso de Psicologia do Instituto em Psicologia da UERJ.

$* * * * *$ Discente no curso de psicologia do Instituto em Psicologia da UERJ .

$* * * * * *$ Mestranda do Programa de Pós-Graduação em Psicologia da UERJ .

******* Mestranda do Programa de Pós-Graduação em Psicologia da UERJ .

$* * * * * * * *$ Discente no curso de psicologia do Instituto de Psicologia da UERJ .

$* * * * * * * * *$ Doutoranda do Programa de Pós-Graduação em Psicologia da UERJ .

$* * * * * * * * * *$ Discente no curso de Psicologia do Instituto em Psicologia da UERJ .

$* * * * * * * * * * *$ Discente no curso de Psicologia do Instituto de Psicologia da UERJ .

$* * * * * * * * * * * *$ Doutoranda do Programa de Pós-Graduação em Psicologia da UERJ .

$* * * * * * * * * * * * *$ Mestranda do Programa de Pós-Graduação em Psicologia da UERJ .

Este artigo de revista Estudos e Pesquisas em Psicologia é licenciado sob uma Licença Creative Commons Atribuição-Não Comercial 3.0 Não Adaptada. 